\title{
Impact of alcohol additives concentration on etch rate and surface morphology of (100) and (110) Si substrates etched in KOH solutions
}

\author{
Krzysztof P. Rola $\cdot$ Irena Zubel
}

Received: 6 July 2012/ Accepted: 21 September 2012/Published online: 6 October 2012

(c) The Author(s) 2012. This article is published with open access at Springerlink.com

\begin{abstract}
The influence of alcohol concentration on etch rate and surface morphology of (100) and (110) Si planes was investigated in this paper. The etching processes were carried out in $\mathrm{KOH}$ solutions with different concentrations of isopropanol and butanols. The etch rate minima versus alcohol concentration were observed for all the alcohols used in the experiments. Furthermore, close to the concentrations of etch rate minima, the smooth (110) planes were obtained. However, the (100) surfaces were covered with hillocks at these concentrations. Based on the surface tension measurements and literature reports, the explanation of appearance of the etch rate minimum was suggested. In the proposed model, the adsorption maximum corresponds to the complete formation of an alcohol monolayer on Si surface and, consequently, to the etch rate minimum. At higher concentrations of alcohol, the monolayer disappears and the etch rate increases.
\end{abstract}

\section{Introduction}

Micromachining of monocrystalline silicon by anisotropic wet chemical etching is a well-known technique for fabrication of three-dimensional MEMS and MOEMS (optical MEMS) structures (Hoffmann and Voges 2002; Pal and Sato 2009, 2010). In the field of MOEMS, manufacturing of micromirrors formed by $\{h k l\}$ sidewall planes is particularly interesting (Sadlery et al. 1997; Resnik et al. 2000; Strandman et al. 1995), due to the exact geometries of the

K. P. Rola $(\bowtie) \cdot$ I. Zubel

Faculty of Microsystem Electronics and Photonics,

Wrocław University of Technology, Janiszewskiego 11/17,

50-372 Wrocław, Poland

e-mail: krzysztof.rola@pwr.wroc.pl etched microstructures and the simplicity of the wet etching technology. The $\{110\}$ planes are especially attractive, because they can serve as micromirrors inclined at $45^{\circ}$ towards the (100) substrate and thus reflect a light beam at an angle of $90^{\circ}$ (Strandman et al. 1995; Resnik et al. 2005; Yagyu et al. 2010; Xu et al. 2011). However, to be useful as micromirrors, the $\{110\}$ planes ought to be very smooth. Moreover, if a light beam is emitted from an optical fiber aligned in a groove fabricated in (100) substrate, the quality of the (100) surface is also important. The large hillocks covering the (100) surface after anisotropic etching could make the appropriate alignment of optical fiber on (100) surface difficult.

Many studies have been done on surface morphologies and etch rates of (100) and (110) Si planes so far (Bressers et al. 1996; Sato et al. 1999; Zubel et al. 2001; Zubel and Kramkowska 2001; van Veenendaal et al. 2001; Gosalvez and Nieminen 2003; Cho Wen-June et al. 2004; Cheng et al. 2006). The etching processes were usually carried out in TMAH or $\mathrm{KOH}$ aqueous solutions, optionally with addition of surfactants or alcohols. The $\mathrm{KOH}$ solution saturated with isopropyl alcohol (also called isopropanol or IPA) was often used, because it provides high etch rate ratio $\mathrm{R}(100) / \mathrm{R}(110)$, smooth (100) surface and reduction of convex corner undercut (Backlund and Rosengren 1992; Zubel 2001; Zubel and Kramkowska 2004). However, the $\{110\}$ planes are patterned with stripes after etching in this solution (Backlund and Rosengren 1992; Zubel and Kramkowska 2004). Although smooth $\{110\}$ planes can be obtained in TMAH-based solution (Resnik et al. 2005; Yagyu et al. 2010; Xu et al. 2011), it is quite expensive and usually provides lower etch rates than $\mathrm{KOH}$. Therefore, there is still a need for searching for the composition of the $\mathrm{KOH}$-based solution in which smooth $\{110\}$ planes are achievable. 
One of the ways of modifying the etching solution is changing a concentration of alcohol additive. The previous studies in the literature were rarely focused on the etching in $\mathrm{KOH}$ solutions with isopropanol concentration below the saturation level. This is mainly because the $\mathrm{KOH}$ solution saturated with isopropanol provides the smoothest (100) surface and maintaining the constant alcohol concentration of the saturated solution is much easier. Nevertheless, the relatively smooth (110) plane has been obtained at the concentration of isopropyl alcohol distinctly below the saturation level, recently (Zubel et al. 2011). Moreover, the minimum of the etch rate has been observed at this concentration.

In this work, we investigate if the similar phenomena occur in the $\mathrm{KOH}$ solutions with addition of other alcohols. The previous reports showed that the addition of butyl alcohols to the etching solution results in a high $\mathrm{R}(100) / \mathrm{R}(h k l)$ etch rate ratio and a large reduction of convex corner undercut (Zubel and Kramkowska 2002; Rola and Zubel 2011). Therefore, in this paper, we study in detail the influence of butyl alcohols concentrations on surface morphologies and etch rates of (100) and (110) Si planes. The results of etching in $\mathrm{KOH}$ solution with isopropanol are also presented. To examine the mechanism of behavior of alcohol molecules in the etching solution, the measurements of surface tension of the solutions are carried out, too.

\section{Results and discussion}

\subsection{Experimental details}

Silicon wafers of (100) and (110) orientations were used in the experiments. The etching processes of the wafers were carried out in $\mathrm{KOH}$ aqueous solutions with addition of alcohols at different concentrations. The alcohol concentration was increased up to the saturation level, which could be observed as a separate alcohol layer on the solution surface. Isopropanol, 1-butanol, isobutanol and tert-butanol were used as the additives (Fig. 1). The $\mathrm{KOH}$ concentration was 3 or $5 \mathrm{M}$ ( 15 or $23 \mathrm{wt} \%)$. The etching experiments were conducted in a glass vessel with reflux condenser in stirred conditions (mechanical agitation at $210 \mathrm{rpm}$ ). The wafers were etched for $60 \mathrm{~min}$., at temperature of $75 \pm 0.5^{\circ} \mathrm{C}$.

The surface morphologies of the etched $\mathrm{Si}$ substrates were evaluated by SEM. The etch rates of the wafers were estimated by the etching depth measurements with a micrometric tool. The surface tensions of the etching solutions were measured by du Noüy ring method with the tensiometer Easydyne by KRÜSS. The measurements were carried out at the room temperature $\left(20^{\circ} \mathrm{C}\right)$, since the measuring vessel had to be open and, thus, the alcohol could evaporate at elevated temperatures.

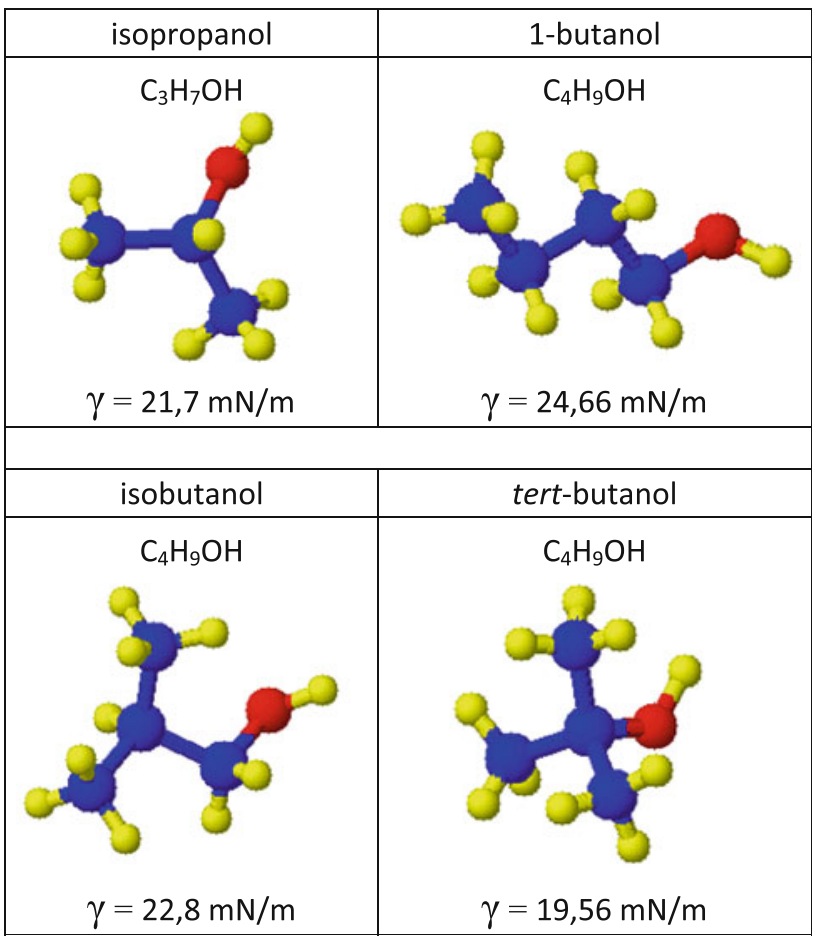

Fig. 1 The schematic structures and surface tensions $\gamma$ of alcohol molecules



Fig. 2 Concentration dependence of (100) and (110) planes etch rates in 3 and $5 \mathrm{M} \mathrm{KOH}$ solutions with isopropanol (Zubel et al. 2011)

\subsection{Dependence of etch rates on alcohol concentration}

The influence of alcohol concentration on etch rates of (100) and (110) planes is shown in Figs. 2, 3, 4, 5. In case of all investigated alcohols, the addition of a small amount of alcohol to the $\mathrm{KOH}$ solution results in significant reduction of the etch rates of both planes. At certain concentrations of the alcohols, the minima of the etch rates of (100) and (110) planes can be observed. Further increase of 


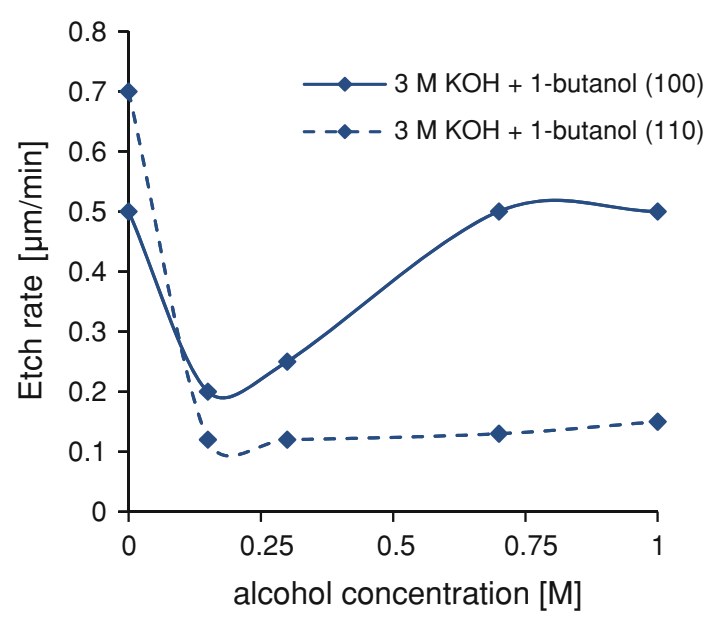

Fig. 3 Concentration dependence of (100) and (110) planes etch rates in $3 \mathrm{M} \mathrm{KOH}$ solutions with 1-butanol

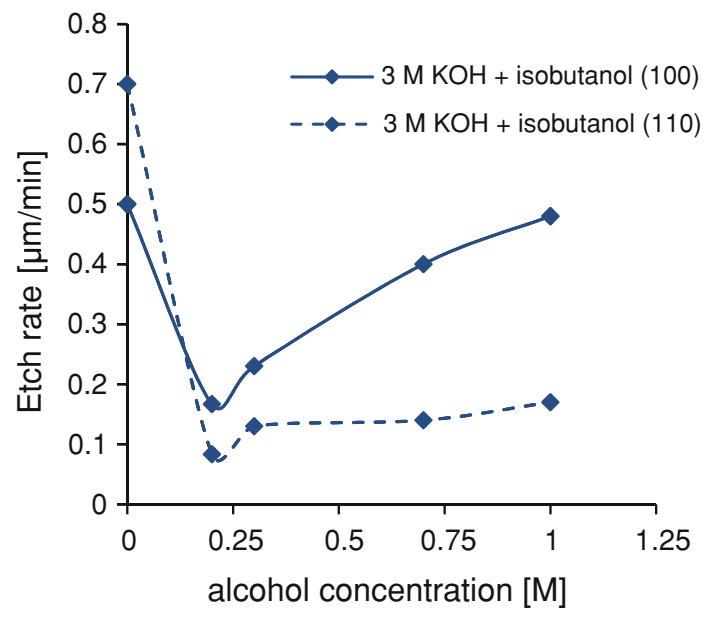

Fig. 4 Concentration dependence of (100) and (110) planes etch rates in $3 \mathrm{M} \mathrm{KOH}$ solutions with isobutanol

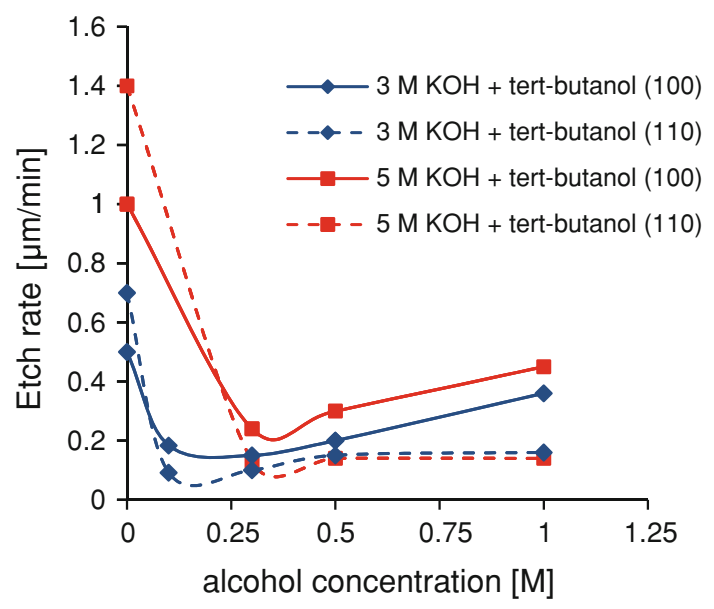

Fig. 5 Concentration dependence of (100) and (110) planes etch rates in 3 and $5 \mathrm{M} \mathrm{KOH}$ solutions with tert-butanol alcohols concentrations causes increment of the etch rates until the saturation level. The phenomenon is especially visible for (100) planes.

The appearance of the minima on etch rate curves versus alcohol concentration is difficult to explain. It is well known that saturation of the $\mathrm{KOH}$ solution with alcohol considerably reduces the (110) etch rate whereas the reduction of (100) etch rate is relatively low (Zubel et al. 2001; Zubel and Kramkowska 2004). The effect could be explained by preferential adsorption of alcohol molecules on the (110) plane. The adsorbed molecules are believed to inhibit the access of reactants $\left(\mathrm{H}_{2} \mathrm{O}\right.$ and $\mathrm{OH}^{-}$groups) to the $\mathrm{Si}$ surface, which results in decrement of the etch rate. Thus, the gradual increase in alcohol concentration should lead to the stronger adsorption of the alcohol molecules and larger reduction of the etch rate. Nevertheless, this explanation is not sufficient to describe the occurrence of the etch rates minima of (110) as well as (100) planes.

\subsection{Surface morphology versus alcohol concentration}

Although saturation of the $\mathrm{KOH}$ solution with isopropyl alcohol causes disappearance of hillocks on (100) Si surface, it does not improve significantly the roughness of (110) plane, which is patterned with stripes (Fig. 6). Figure 7 shows the surface morphologies of (100) and (110) substrates which were etched in the $\mathrm{KOH}$ solutions with different IPA concentrations. The increment of the alcohol concentration gradually reduces the density of hillocks covering the (100) surface. However, the effect of the alcohol concentration on the (110) plane is quite opposite, because the lowest roughness of it occurs at a low (1 M) concentration of isopropanol. Moreover, the minimum of the (110) etch rate appears at this concentration (Fig. 2).

The similar phenomena are observed for butyl alcohols (Figs. 8, 9, 10). The smooth (110) surfaces are obtained at $0.2 \mathrm{M}$ of isobutanol and $0.1-0.3 \mathrm{M}$ of tert-butanol, which are the concentrations of their etch rate minima. The (100) planes are covered with hillocks at these concentrations, too. Nevertheless, the hillocks are relatively small (especially in the case of isobutanol) as compared to those etched in the solution with $1 \mathrm{M}$ of isopropanol.

Increasing isobutyl and tert-butyl alcohol concentrations up to 0.3 and $0.55 \mathrm{M}$, respectively, results in appearing of large hillocks. When the $\mathrm{KOH}$ solution becomes saturated with isobutanol, the density of hillocks on the (100) surface decreases. The hillocks almost completely disappear from the (100) surface when the solution becomes saturated with 1-butanol. However, the smooth (110) surface is not obtained at any concentration of 1-butanol, though its best quality is achieved in the non-saturated solution.

The etch rate ratio $R(100) / R(110)>1$ is maintained at the alcohols concentrations of etch rate minima, which 


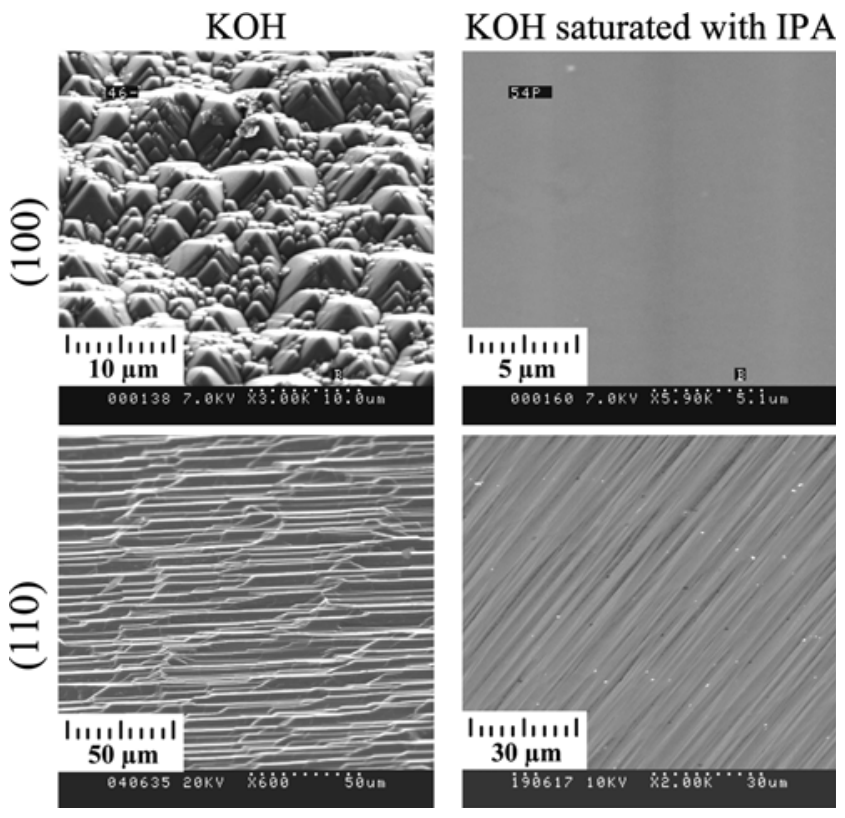

Fig. 6 SEM images of (100) and (110) surfaces etched in pure $3 \mathrm{M}$ $\mathrm{KOH}$ solution and in $3 \mathrm{M} \mathrm{KOH}$ solution saturated with isopropanol

means that $\{110\}$ planes would develop in the sidewalls in $\langle 100\rangle$ directions in the (100) substrate. The $\{110\}$ sidewalls could form micromirrors inclined at $45^{\circ}$ towards the (100) bottom. Although the (100) surface is rough at the concentrations of etch rate minima, the presence of tiny hillocks in the solutions with isobutanol and tert-butanol could be negligible for optical fibers alignment. In the Fig. 11, the $45^{\circ}$ micromirrors fabricated in the $\mathrm{KOH}$ solution with addition of $0.1 \mathrm{M}$ of tert-butyl alcohol are compared with those obtained in the $\mathrm{KOH}$ solution saturated with IPA. In contrast to the striped pattern of the $\{110\}$ sidewalls after etching with isopropanol addition, the smooth mirror-like $\{110\}$ planes can be achieved in the solution with the low concentration of the butyl alcohol. Nevertheless, from the practical point of view, maintaining such small amounts of alcohol in the solution during a long etching process at the elevated temperature would be difficult due to the alcohol evaporation.

\subsection{Discussion based on surface tension measurements}

To understand the surface related phenomena occurring in the $\mathrm{KOH}$ aqueous solutions containing alcohol additives at different concentrations, it seems to be necessary to analyze what generally happens in the water-based solutions as alcohol concentration changes. Although, to our best knowledge, there is no significant report on the aqueous solutions containing both electrolyte (e.g., $\mathrm{KOH}$ or $\mathrm{NaOH}$ ) and alcohol, the aqueous solutions with alcohols have been widely studied in the literature (Zana and Michels 1989; Hayashi et al. 1990; D’Angelo et al. 1994; Gliński et al. 1998; Yoshida et al. 2002; Yano 2005). Based on these reports, the phenomena concerning alcohol molecules behavior in the aqueous solution at different alcohol concentrations are described below.

It is believed that three main factors determine the mixing state of alcohol aqueous solutions, i.e., hydrogen bonding, hydrophobic interaction and entropy contribution
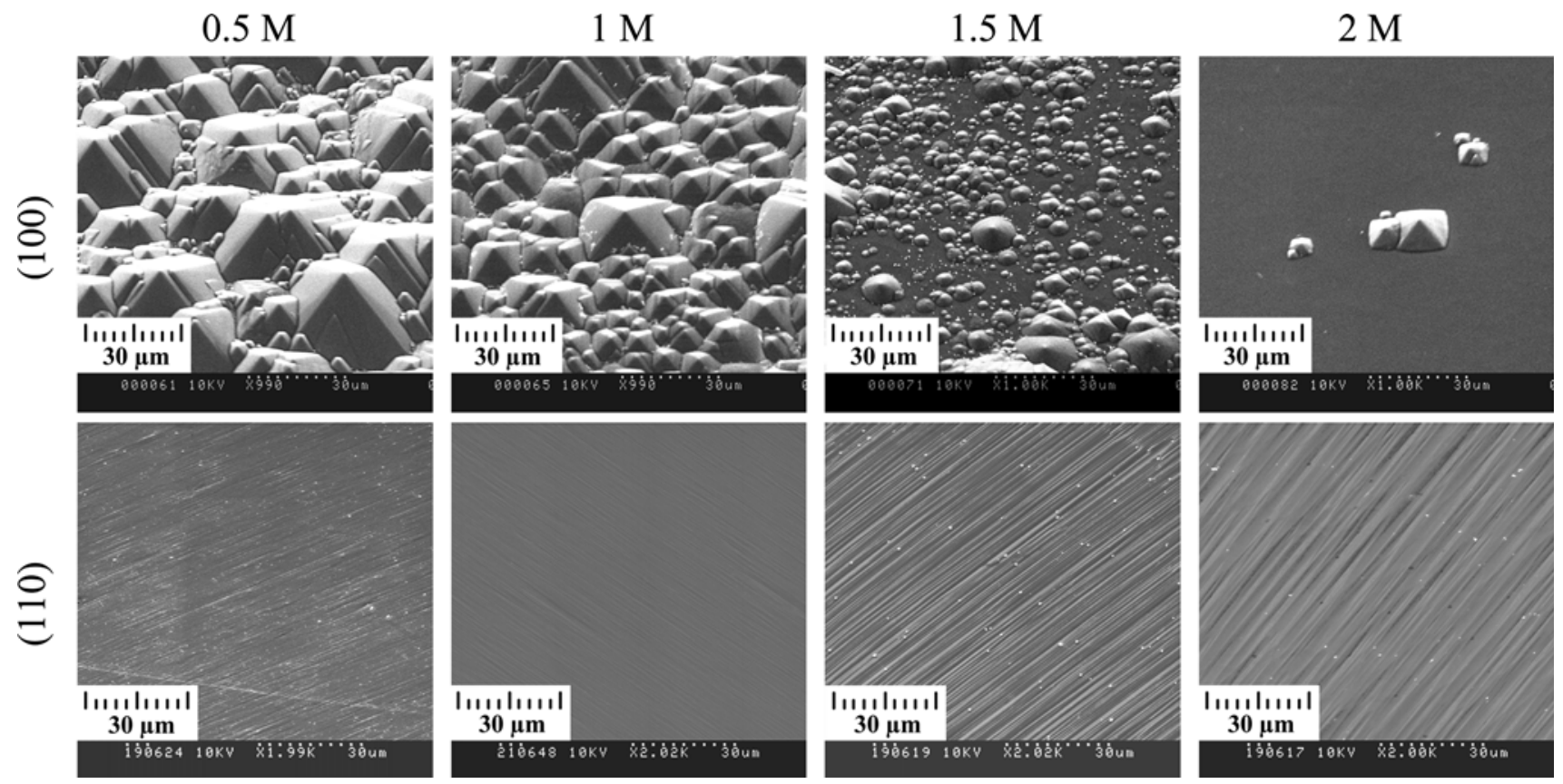

Fig. 7 SEM images of (100) and (110) Si surfaces etched in 3 M KOH solutions with different concentrations of isopropanol (Zubel et al. 2011) 

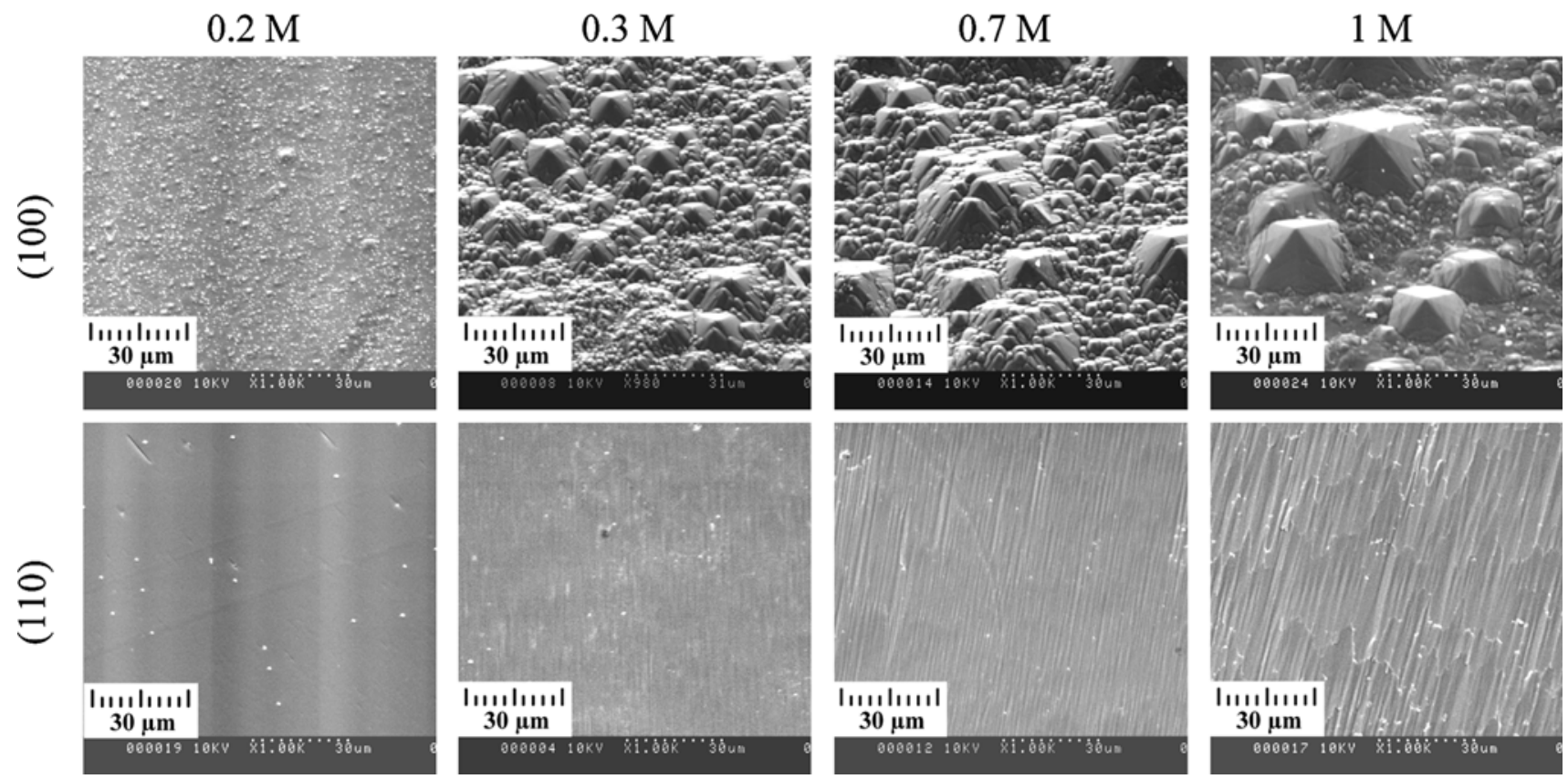

Fig. 8 SEM images of (100) and (110) Si surfaces etched in $3 \mathrm{M} \mathrm{KOH}$ solutions with different concentrations of isobutanol


Fig. 9 SEM images of (100) and (110) Si surfaces etched in $3 \mathrm{M} \mathrm{KOH}$ solutions with different concentrations of tert-butanol

(Hayashi et al. 1990). In a liquid phase, water molecules are not completely disordered and form a kind of network by hydrogen bonds. When an alcohol molecule is inserted into the water network, the hydrogen bond is formed between the hydrophilic hydroxyl group of alcohol and a water molecule, whereas the hydrophobic hydrocarbon group of alcohol interacts with surrounding water molecules with van der Waals forces (Chaplin 2012; Klymkowsky 2012). In this way, the hydrogen-bonding network of water forms cages around alcohol monomers in a diluted alcohol aqueous solution (D'Angelo et al. 1994; Yoshida et al. 2002). This phenomenon, called hydrophobic hydration, makes the liquid structure more ordered than in the case of pure water, which results in an unfavorable entropy decrease (Glinski et al. 1998; Klymkowsky 2012).

Introduction of alcohol molecules into water results not only in modification of the bulk structure of water but also in adsorption of the alcohol molecules at the liquid-air interface. The alcohol molecules are adsorbed so that their carbon chains are oriented toward air and their hydroxyl groups are oriented toward the water solution. As the concentration of alcohol in the aqueous solution increases, more and more alcohol molecules gather at the interface and, as a consequence, the surface tension of the solution goes down. When the concentration of alcohol is sufficiently high, the monolayer of the alcohol molecules at the 

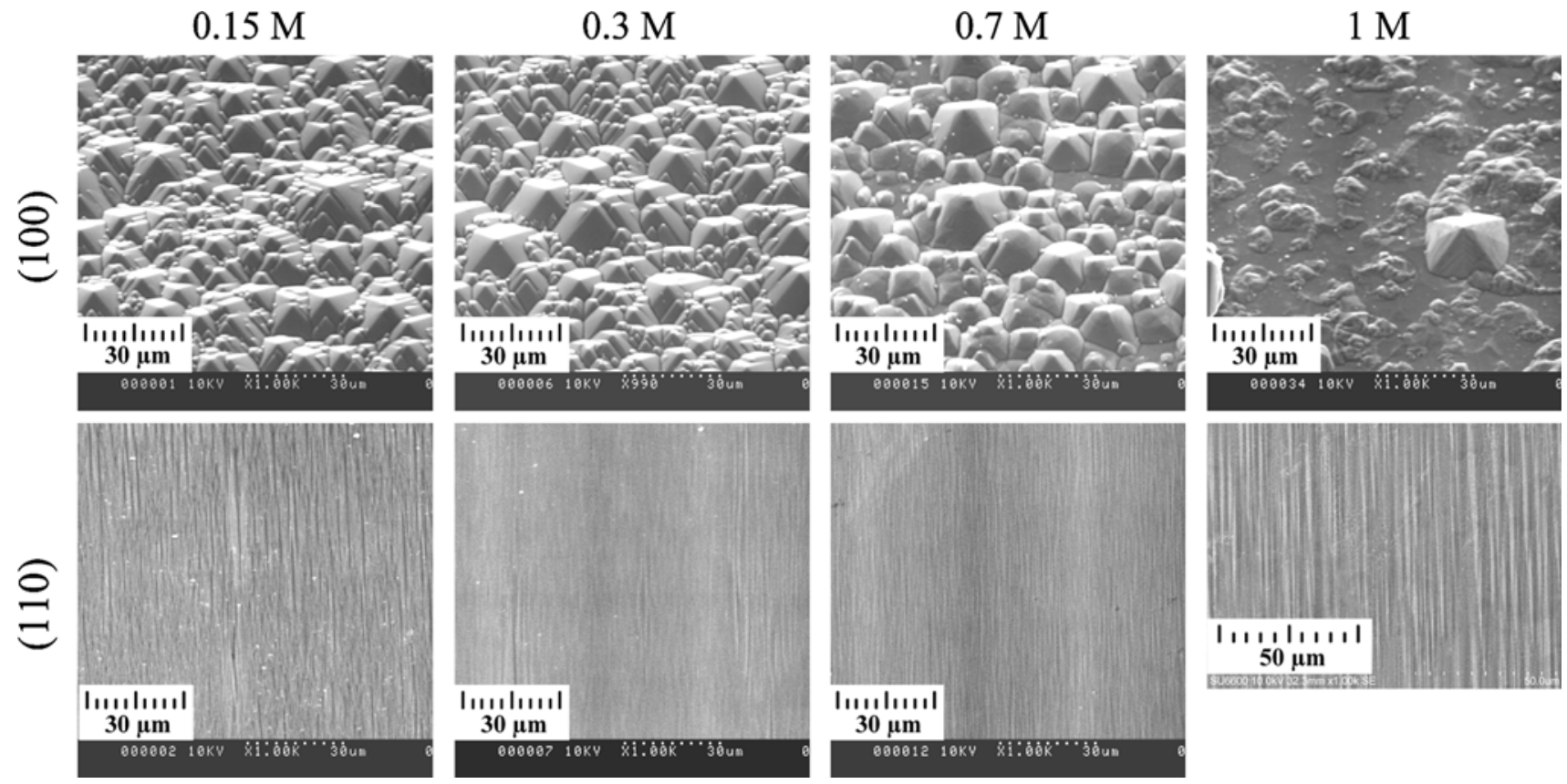

Fig. 10 SEM images of (100) and (110) Si surfaces etched in $3 \mathrm{M} \mathrm{KOH}$ solutions with different concentrations of 1-butanol

Fig. 11 SEM images of $\{110\}$ micromirror sidewalls fabricated in $\operatorname{Si}(100)$ substrate in $3 \mathrm{M} \mathrm{KOH}$ solutions saturated with IPA and with addition of $0.1 \mathrm{M}$ of tert-butanol
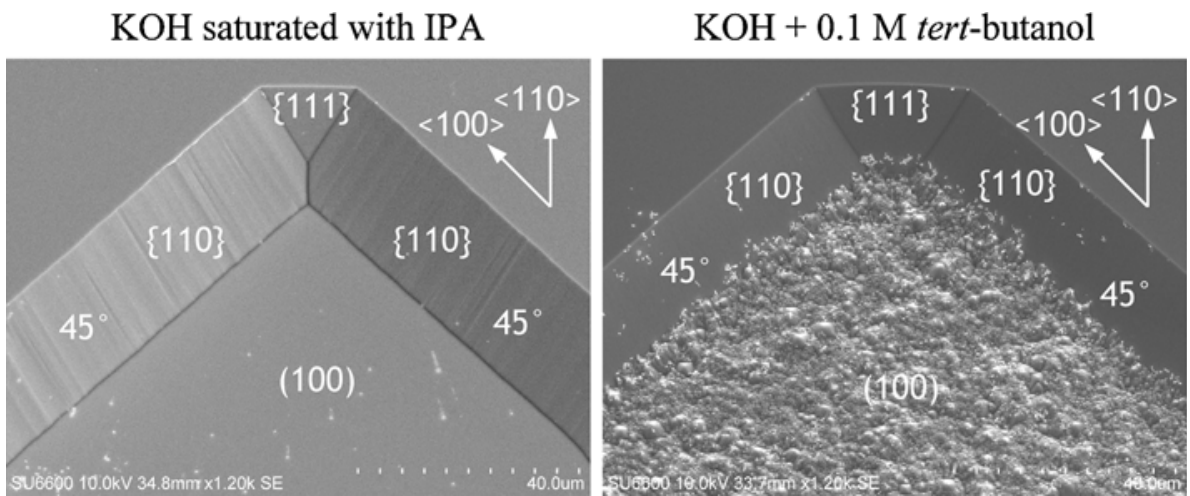

interface is completely formed. The concentration of the monolayer formation corresponds to that at which the solution loses its ideality and the alcohol molecules in the bulk of solution start to aggregate (Yano 2005). The aggregation occurs due to the hydrophobic attraction between hydrocarbon groups of alcohol molecules and is favorable because requires less entropy reduction than the hydrophobic hydration of single molecules of alcohol (Glinski et al. 1998; Yoshida et al. 2002). The hydroxyl groups of the aggregated molecules are oriented towards the aqueous phase so that they form hydrogen bonds with the surrounding water molecules.

Further increase of the alcohol concentration leads to the breakage of hydrogen bond network by the aggregates of alcohol molecules and weakening of van der Waals interactions in the bulk (D'Angelo et al. 1994; Yoshida et al. 2002; Yano 2005). As a consequence, the alcohol molecules move from the liquid-air interface into the bulk, causing a gradual disappearance of the alcohol monolayer (Yano 2005). In the high concentrated solution, water molecules are incorporated as single molecules into the alcohol hydrogen-bonding structure (D'Angelo et al. 1994; Yoshida et al. 2002; Czarnecki and Wojtków 2008).

The above mentioned considerations do not take into account the saturation of aqueous solution with alcohol. Although both isomers of propyl alcohol are completely soluble in water, butanols, except for tert-butanol, have limited water solubility. Thus at some butanol concentrations, aqueous solutions become saturated with them and a phase separation between alcohol and water occurs. According to the study on 1-butanol made by Zana and Michels (1989), the solution saturation with alcohol appears slightly above the concentration at which the alcohol molecules start to aggregate.

Since the addition of an electrolyte (such as $\mathrm{KOH}$ ) to the solution reduces the alcohol solubility in water, the 


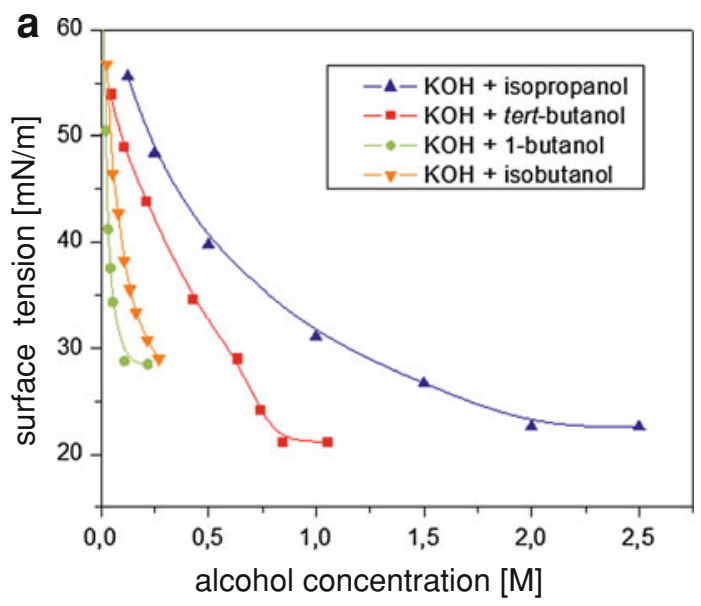

b

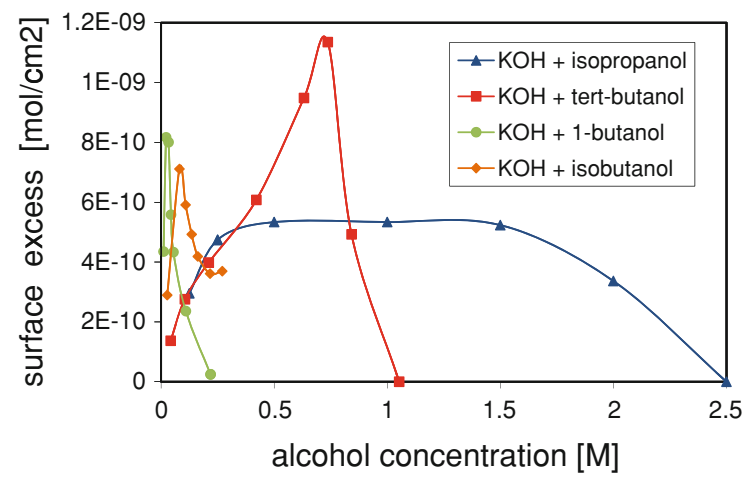

Fig. 12 a Surface tension and b surface excess as a function of alcohol concentration in 3 M KOH aqueous solutions (Rola and Zubel 2011)

saturation occurs in all the $\mathrm{KOH}$ aqueous solutions with alcohols used in the experiments. Figure 12a shows the dependence of surface tension on alcohol concentration in $3 \mathrm{M} \mathrm{KOH}$ solutions containing different propyl and butyl alcohols. As it could be expected, surface tension decreases as the alcohol concentration increases, until the saturation level is reached. Based on the results of surface tension measurements, surface excess was calculated (Fig. 12b), using the Gibbs adsorption equation (1):

$\Gamma=-\frac{c}{R T} \cdot \frac{d \sigma}{d c}$

where $\Gamma$ is the surface excess, $c$ is the solution concentration, $\sigma$ is the surface tension, $T$ is the temperature $\left(20{ }^{\circ} \mathrm{C}\right.$ ) and $R$ is the gas constant. Surface excess is the difference between the concentration of solute (e.g., alcohol) at the interface and its concentration in the bulk of solution. If the solute concentration at the interface is distinctly higher than in the bulk, the surface excess gives information about adsorption density.

As the concentrations of alcohols in the $\mathrm{KOH}$ solutions increase, the surface excess increases, too (see Fig. 12b). However, after reaching the maximum of adsorption density, the surface excess begins to decrease. The phenomenon occurs more rapidly for butanols than for isopropanol and appears at the lower concentrations in the case of less branched butanols (compare Figs. 1, 12b). Despite these differences, the results suggest that after a complete formation of alcohol monolayer, which corresponds to the adsorption maxima in the Fig. 12b, the continued increase in alcohol concentration leads to the gradual disappearance of the alcohol monolayer. Obviously it cannot be concluded that the monolayer disappears at all, since the zero values of surface excess in Fig. 12b result from the saturation of the solution. When the solution becomes saturated with alcohol, the increase in alcohol concentration does not change the surface tension and, as a consequence, the surface excess is zero. Nevertheless, as it was shown by Zana and Michels (1989), there is a short range of alcohol concentration between the formation of monolayer and saturation of the solution, in which the density of molecules in the monolayer could decrease. The decrease of surface excess can be also related to the disappearance of the alcohol monolayer caused by the breakage of hydrogen bond network in the water-alcohol mixtures.

During anisotropic etching of silicon the alcohol molecules are believed to be adsorbed not only at the airsolution interface but also on the Si surface. The silicon surface is supposed to be H-terminated in the etching solution, which makes the surface hydrophobic (Wind and Hines 2000). Thus, the alcohol molecules are adsorbed on the Si surface with their hydrocarbon chains by van der Waals forces whereas their hydroxyl groups are oriented towards the bulk of solution. The adsorbed molecules inhibit access of the reactants and consequently reduce the etch rate. Presuming that analogous phenomena take place at the air-solution interface and on the silicon surface, we propose the possible mechanism of alcohol molecules interaction with the $\mathrm{Si}$ surface depending on the alcohol concentration.

When the alcohol concentration in the etching solution is low, the increase in alcohol concentration results in a denser adsorption of the alcohol molecules on the Si surface and consequently the larger reduction of etch rate. When the alcohol molecules start to aggregate and the alcohol monolayer on the Si surface is completely formed, the etch rate reaches its minimum. Further increase of the alcohol concentration causes the breakage of hydrogen bond network by the alcohol aggregates. As a result, the monolayer begins to disappear and the etch rate increases. This process continues until the solution becomes saturated with alcohol (see Fig. 13). 


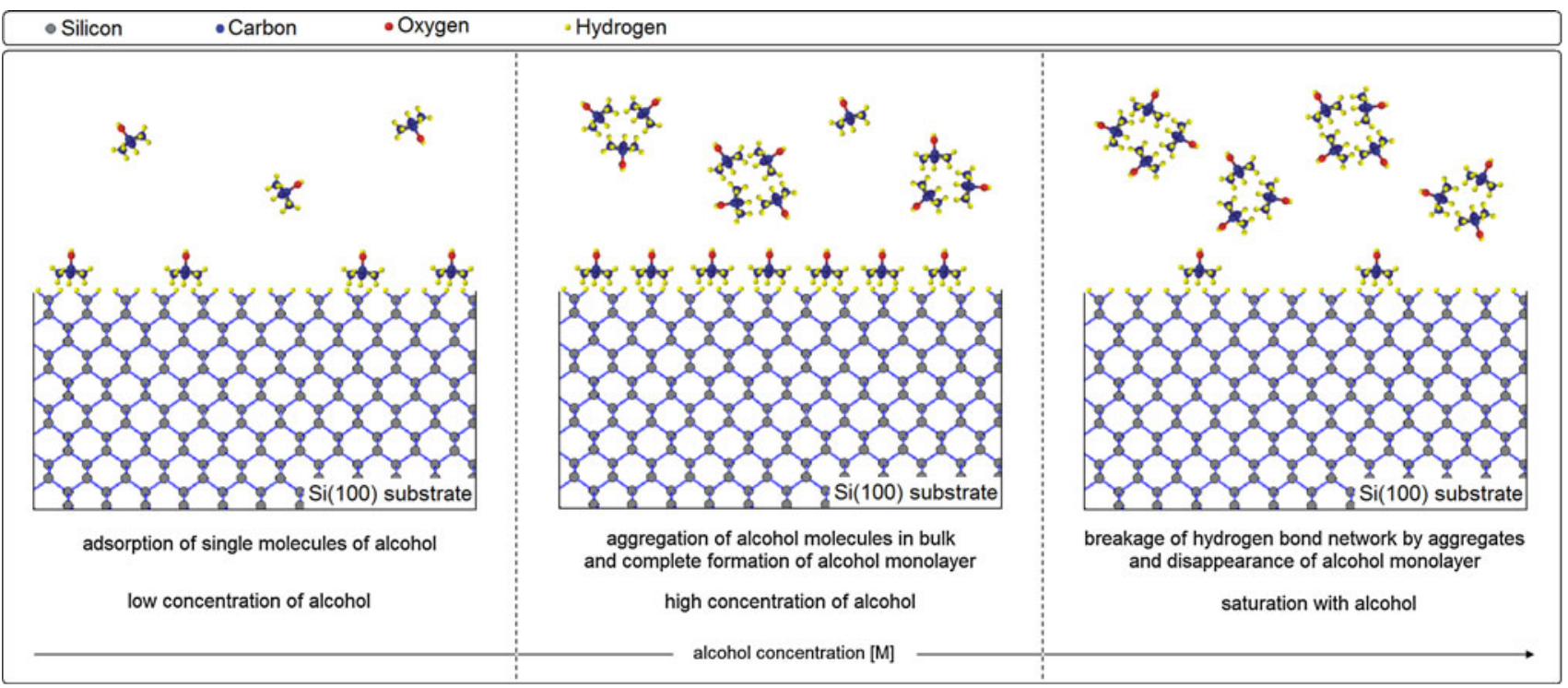

Fig. 13 Simplified model of alcohol molecules behavior at various alcohol concentrations during anisotropic etching of (100) silicon

The etch rate of the (100) plane increases much more than that of the (110) one as the alcohol concentration increases. This suggests that adsorption on the (110) surface is stronger and even the breakage of hydrogen bond network does not considerably diminish the density of alcohol molecules on the (110) surface. The stronger alcohol adsorption on the (110) surface than on the (100) surface is probably somehow connected with the difference in the surface bonds configuration between both planes. Perhaps, the (110) surface is more hydrophobic which makes the alcohol molecules more likely to adsorb on it. Therefore it seems that the research on the wetting characteristics of the differently oriented silicon wafers should be conducted to explain the difference between both planes.

The large increase in the (100) etch rate when the solution becomes saturated could also result from the decrease in the density of hillocks covering the (100) surface, since the hillocks are bounded by the slow etching $\{111\}$ planes and slow the etching process of (100) surface to some degree. However, this might also be explained from the point of view of the change of adsorption density versus alcohol concentration. If the adsorbed alcohol molecules are the micromasks for hillocks formation (Rola and Zubel 2011), the denser adsorption of alcohol molecules should also cause the denser distribution of hillocks and, as a consequence, the larger reduction of the (100) etch rate.

\section{Conclusions}

The influence of alcohol concentration in $\mathrm{KOH}$ aqueous solution on etch rate and surface morphology of (100) and (110) planes was studied in this paper. Isopropanol and three isomers of butanols were used in the experiments. The minima of the etch rates versus alcohol concentration were observed not only for isopropanol but also for butanols. Furthermore, the roughness of the (110) surfaces was reduced at the concentrations close to the occurrence of the etch rate minima. Although the (100) surfaces were covered with hillocks at these concentrations, the hillocks appearing on the surfaces etched in the solutions with low concentrations of isobutanol and tert-butanol were relatively small. Generally, saturation of the $\mathrm{KOH}$ solutions with the alcohols resulted in the decrease in hillocks density on the (100) surface, though the intensity of the decrease was different depending on the alcohol.

Due to the little sizes of the hillocks, the low roughness of (110) planes, and the etch rate ratio $\mathrm{R}(100) / \mathrm{R}(110)>1$, the solutions with low concentrations of isobutanol and tert-butanol could be used for fabrication of the $\{110\}$ micromirrors inclined at $45^{\circ}$ towards the (100) substrate. The smooth $45^{\circ}$ micromirrors were actually obtained in the $\mathrm{KOH}$ solution with addition of $0.1 \mathrm{M}$ of tert-butanol, which is advantage over the popular $\mathrm{KOH}$ solution saturated with IPA, which yields rough $\{110\}$ sidewalls. Nonetheless, maintaining such low concentration of the alcohol during long etching processes at the elevated temperature would be difficult because of the alcohol evaporation, which is kind of a limitation of the method.

Based on the etch rate results, surface tension measurements and literature reports, the mechanism of alcohol interaction with silicon surface during anisotropic etching was proposed. It is supposed that the minimum of the etch rate versus the alcohol concentration corresponds to the maximum density of alcohol molecules adsorbed on the $\mathrm{Si}$ surface. When the alcohol concentration becomes higher, the hydrogen bond network is gradually broken, which 
results in the increase in the etch rate. The phenomenon occurs until the saturation of the etching solution with alcohol. The etch rate minima are much more visible for the (100) plane than for the (110) one, which may result from the higher hydrophobicity of the latter one and, as a consequence, the stronger adsorption of alcohol molecules on it. However, this assumption should be proved by wettability measurements of $\mathrm{Si}$ wafers with different crystallographic orientations.

Acknowledgments This work was co-financed by Polish Ministry of Science and Higher Education under the grant no. N N515 497140, by the European Union within European Regional Development Fund, through grant Innovative Economy (POIG.01.01.02-00-008/08$05)$ and by Wroclaw University of Technology statutory grant S20010, B20011.

Open Access This article is distributed under the terms of the Creative Commons Attribution License which permits any use, distribution, and reproduction in any medium, provided the original author(s) and the source are credited.

\section{References}

Backlund Y, Rosengren L (1992) New shapes in (100) Si using KOH and EDP etches. J Micromech Microeng 2:75-79

Bressers PMMC, Kelly JJ, Gardeniers JCE, Elwenspoek M (1996) Surface morphology of p-type (100) silicon etched in aqueous alkaline solution. J Electrochem Soc 143:1744-1750

Chaplin M (2012) Hydrophobic hydration. Water structure and science. http://www.btinternet.com/ martin.chaplin/phobic.html

Cheng D, Gosalvez MA, Hori T, Sato K, Shikida M (2006) Improvement in smoothness of anisotropically etched silicon surfaces: effects of surfactant and TMAH concentrations. Sens Actuators A 125:415-421

Czarnecki MA, Wojtków D (2008) Effect of varying water content on the structure of butyl alcohol/water mixtures: FT-NIR twodimensional correlation and chemometric studies. J Mol Struct 883-884:203-208

D’Angelo M, Onori G, Santucci A (1994) Self-association behaviour of alcohols in diluted aqueous solutions. Il Nuovo Cimento D 16:1499-1514

Gliński J, Chavepeyer G, Platten J-K, Smet P (1998) Surface properties of diluted aqueous solutions of normal short-chained alcohols. J Chem Phys 109:5050

Gosalvez MA, Nieminen RM (2003) Surface morphology during anisotropic wet chemical etching of crystalline silicon. J Phys 5:100.1-100.28

Hayashi H, Nishikawa K, Iijima T (1990) Small-angle x-ray scattering study of fluctuations in 1-propanol-water and 2-propanol-water systems. J Phys Chem 94:8334-8338

Hoffmann M, Voges E (2002) Bulk silicon micromachining for MEMS in optical communication systems. J Micromech Microeng 12:349-360

Klymkowsky M (2012) Water, chemical bonds and macromolecular structure. Biofundamentals. Virtual laboratory, University of Colorado. http://www.virtuallaboratory.colorado.edu/Biofundamentals/ lectureNotes/Topic2AB_Water.html

Pal P, Sato K (2009) Complex three-dimensional structures in $\mathrm{Si}$ $\{100\}$ using wet bulk micromachining. J Micromech Microeng 19:105008
Pal P, Sato K (2010) Fabrication methods based on wet etching process for the realization of silicon MEMS structures with new shapes. Microsyst Technol 16:1165-1174

Resnik D, Vrtacnik D, Amon S (2000) Morphological study of 311 crystal planes anisotropically etched in (100) silicon: role of etchants and etching parameters. J Micromech Microeng 10:430-439

Resnik D, Vrtacnik D, Aljancic U, Mozek M, Amon S (2005) The role of Triton surfactant in anisotropic etching of 110 reflective planes on (100) silicon. J Micromech Microeng 15:1174-1183

Rola KP, Zubel I (2011) Investigation of Si $(h k l)$ surfaces etched in $\mathrm{KOH}$ solutions saturated with tert-butyl alcohol. J Micromech Microeng 21:115026

Sadler DJ, Garter MJ, Ahn CH, Koh S, Cook AL (1997) Optical reflectivity of micromachined $\{111\}$-oriented silicon mirrors for optical input-output couplers. J Micromech Microeng 7:263-269

Sato K, Shikida M, Yamashiro T, Tsunekawa M, Ito S (1999) Roughening of single-crystal silicon surface etched by $\mathrm{KOH}$ water solution. Sens Actuators A 73:122-130

Strandman C, Rosengren L, Elderstig HGA, Backlund Y (1995) Fabrication of $45^{\circ}$ mirrors together with well-defined v-grooves using wet anisotropic etching of silicon. J Microelectromech Syst 4:213-219

van Veenendaal E, Sato K, Shikida M, van Suchtelen J (2001) Micromorphology of single crystalline silicon surfaces during anisotropic wet chemical etching in $\mathrm{KOH}$ and TMAH. Sens Actuators A 93:219-231

Wen-June Cho, Wei-Kuo Chin, Ching-Tung Kuo (2004) Effects of alcoholic moderators on anisotropic etching of silicon in aqueous potassium hydroxide solutions. Sens Actuators A 116:357-368

Wind RA, Hines MA (2000) Macroscopic etch anisotropies and microscopic reaction mechanisms: a micromachined structure for the rapid assay of etchant anisotropy. Surf Sci 460:21-38

Xu YW, Michael A, Kwok CY (2011) Formation of ultra-smooth $45^{\circ}$ micromirror on (100) silicon with low concentration tmah and surfactant: techniques for enlarging the truly $45^{\circ}$ portion. Sens Actuators A 166:164-171

Yagyu H, Yamaji T, Nishimura M, Sato K (2010) Forty-five degree micromirror fabrication using silicon anisotropic etching with surfactant-added tetramethylammonium hydroxide solution. Jpn J Appl Phys 49:096503

Yano YF (2005) Correlation between surface and bulk structures of alcohol-water mixtures. J Colloid Interf Sci 284:255-259

Yoshida K, Yamaguchi T, Kovalenko A, Hirata F (2002) Structure of tert-butyl alcohol-water mixtures studied by the RISM theory. J Phys Chem B 106:5042-5049

Zana R, Michels B (1989) Fluorescence probing and ultrasonic absorption study of the self-association of 1-butanol in aqueous solution. J Phys Chem 93:2643-2648

Zubel I (2001) The influence of atomic configuration of $(h k l)$ planes on adsorption processes associated with anisotropic etching of silicon. Sens Actuators A 94:76-86

Zubel I, Kramkowska M (2001) The effect of isopropyl alcohol on etching rate and roughness of (100) Si surface etched in $\mathrm{KOH}$ and TMAH solutions. Sens Actuators A 93:138-147

Zubel I, Kramkowska M (2002) The effect of alcohol additives on etching characteristics in KOH solutions. Sens Actuators A 101:255-261

Zubel I, Kramkowska M (2004) Etch rates and morphology of silicon $(h k l)$ surfaces etched in $\mathrm{KOH}$ and $\mathrm{KOH}$ saturated with isopropanol solutions. Sens Actuators A 115:549-556

Zubel I, Barycka I, Kotowska K, Kramkowska M (2001) Silicon anisotropic etching in alkaline solutions IV The effect of organic and inorganic agents on silicon anisotropic etching process. Sens Actuators A 87:163-171

Zubel I, Rola K, Kramkowska M (2011) The effect of isopropyl alcohol concentration on the etching process of Si-substrates in $\mathrm{KOH}$ solutions. Sens Actuators A 171:436-445 